\title{
Martine WATRELOT \& Michèle HECQUET (dir.), Le compagnon du Tour de France de George Sand
} Lille, Éditions du Conseil scientifique de l'Université Charles-de-Gaulle Lille 3, coll. Travaux et Recherches, 201 pages

\section{Nicolas Adell}

\section{(2) OpenEdition}

1 Journals

\section{Édition électronique}

URL : https://journals.openedition.org/clio/9967

DOI : $10.4000 /$ clio.9967

ISSN : 1777-5299

Éditeur

Belin

\section{Édition imprimée}

Date de publication : 31 décembre 2010

ISBN : 978-2-8107-0098-1

ISSN : $1252-7017$

\section{Référence électronique}

Nicolas Adell, « Martine watrelot \& Michèle hecauet (dir.), Le compagnon du Tour de France de George Sand », Clio. Femmes, Genre, Histoire [En ligne], 32 | 2010, mis en ligne le 31 décembre 2010, consulté le 01 mai 2022. URL : http://journals.openedition.org/clio/9967 ; DOl : https://doi.org/10.4000/clio. 9967

Ce document a été généré automatiquement le 1 mai 2022.

Tous droits réservés 


\section{Martine WATRELOT \& Michèle HECQUET (dir.), Le compagnon du Tour de France de George Sand}

Lille, Éditions du Conseil scientifique de l'Université Charles-de-Gaulle Lille 3, coll. Travaux et Recherches, 201 pages

\section{Nicolas Adell}

1 Pour le bicentenaire conjoint des naissances de George Sand (1804-1876) et d'Agricol Perdiguier (1805-1876), Michèle Hecquet et Martine Watrelot ont eu l'heureuse initiative d'organiser à l'université de Lille 3, en décembre 2005, deux journées d'étude consacrées à l'analyse du Compagnon du Tour de France (1840), roman de G. Sand que l'on sait largement inspiré de la vie d'Agricol Perdiguier, compagnon menuisier, et de son Livre du compagnonnage (1839). Le résultat laisse un sentiment mitigé, celui que l'on éprouve souvent à la lecture d'actes de colloque réunissant des contributions d'intention, d'allure et de qualité différentes et dont il est bien difficile de réaliser l'articulation. Et ce n'est pas le moindre des mérites des éditrices que d'avoir fait en sorte de prévenir le plus possible les inévitables répétitions auxquelles une telle entreprise pouvait prêter. Sans doute les quatorze contributions peuvent-elles ici et là s'exposer à des redondances, mais somme toute celles-ci sont minimes.

2 Un autre défi louable de ce travail éditorial a été de ménager un espace (deux contributions) à des non-universitaires, acteurs du monde compagnonnique, qui ont pu rendre compte de deux approches de l'activité mémorielle des compagnons vis-à-vis de Perdiguier et de son temps: l'une «en acte» présentant les cérémonies de commémoration de la naissance du menuisier et décrivant les usages actuels de son souvenir; l'autre, plus figée, relatant d'une manière générale comment est géré un patrimoine proprement compagnonnique (archives et monuments) qui, pour intéressante qu'elle soit, laisse le lecteur sur sa faim quant au Compagnon $d u$ Tour de France et quant à Perdiguier. Probablement s'agissait-il, par ces interventions qui ouvrent le volume, de signaler l'actualité ou la persistance de l'impact de cette rencontre entre Perdiguier et Sand et la réception « présente » du roman, sa réception 
« en contexte » étant étudiée dans la partie finale de l'ouvrage. L'idée était intéressante mais n'atteint que très imparfaitement son but ; on aurait aimé en lire ici davantage sur l'avenir du roman dans les milieux compagnonniques, sur son destin et son utilisation dans d'autres cercles, sur les effets d'écho auxquels il pouvait se prêter dans les autobiographies ou les romans ouvriers, en bref trouver esquissée quelque chose d'une " esthétique de la réception ». C'est mon regret général qui, en réalité, ouvre aussi sur le constat d'un mérite de cet ouvrage : celui d'inviter à la poursuite d'une recherche et d'encourager à la réflexion. N'est-ce pas là l'essentiel ?

Outre les contributions des deux compagnons qui auraient peut-être trouvé plus justement leur place dans le dernier ensemble, l'« Accueil de l'œuvre ", l'économie de l'ouvrage cherche à recomposer le «monde» du Compagnon du Tour de France selon quatre perspectives : politique, poétique, rapports entre le peuple et l'art, et réception du texte.

4 La dimension politique du texte ne cache pas, dans les quatre contributions qui la mettent en œuvre et particulièrement dans celle de Bruno Viard, que ses véritables héros sont - loin devant Perdiguier et le compagnonnage, et avant même Sand - Pierre Leroux, le libéralisme et l'idée républicaine. Ceux-ci se montrent selon deux modalités distinctes: d'une part, dans le jeu même de l'écriture et du style et, de l'autre, au travers de certaines références (la société secrète, Rousseau) qui sont au départ d'une réflexion sur le politique au sens le plus général du terme. La première modalité est explicitée par Jean-Louis Cabanès qui montre l'importance de la forme comique et plus généralement de la mascarade, des sous-entendus, des doubles sens - l'ensemble formant ce qu'il appelle le "fictionnaire» (p.32) - qui constituent la condition d'expression du politique dans le roman. L'analyse aurait sans doute acquis une force supplémentaire si elle avait pu montrer les relations que le « fictionnaire » entretient avec l'épaississement général du monde qui a lieu à partir de la modernité révolutionnaire - songeons au retour des tropes, à la (re)découverte des rites initiatiques, au feuilletage mythique des nations, à l'allégorisation de la vie sociale et politique mis en lumière dans les travaux de Maurice Agulhon. De plus, la mise en regard du « fictionnaire » et de « l'effet de réel » cher à Roland Barthes aurait permis de cerner les contours exacts de son champ d'application.

5 La seconde modalité d'évocation du politique est d'abord exprimée par la référence que le Compagnon $d u$ Tour de France fait aux sociétés secrètes, non seulement aux compagnonnages, mais également aux carbonari. Deux approches, bien différentes mais qui visent au même résultat, sont ici représentées: la première ( 0 . Bara) voit dans l'organisation de la société secrète en général, et du compagnonnage en particulier, la formule empêchant le repli sur soi et générant de ce fait le contre-individualisme indispensable à l'établissement d'un socialisme éclairé qui serait l'objet de l'éducation politique du Compagnon. Or, les sociétés compagnonniques offrent justement un magnifique contre-exemple à cette approche générale: des projets personnels, des stratégies individualistes peuvent s'y développer et y sont tôt encouragés par certains usages (dont les moindres ne sont pas l'autobiographie et la réalisation personnelle du chef-d'œuvre). Il reste que la société secrète, comme le salon, le cercle, le club et toutes les autres communautés "resserrées", constitue, ainsi que le montre parfaitement Michèle Hecquet dans sa belle et riche contribution, l'espace privilégié d'un apprentissage de la République et de ses idées. 
6 La figure de Rousseau qu'Olivier Bara repère avec raison dans le roman de Sand est la seconde référence de l'approche politique: Pierre Huguenin, le héros de Sand, est menuisier comme Perdiguier mais surtout, comme Émile Leroux, le modèle politique de Sand, il est tout à fait rousseauiste jusque dans son souci de la " perfectibilité » dont on sait, mais cela aurait pu être davantage souligné par Bruno Viard, qu'elle est l'une des vertus qui, selon Rousseau, distingue l'homme du reste du vivant.

7 La partie «Poétique » du volume est importante et englobe, de fait, non seulement les contributions regroupées sous l'intitulé «Poétique » mais également celles mises sous le patronage «Le peuple et l'art». Est-ce dans le souci d'éviter un déséquilibre trop important de la structure de l'ouvrage que ces parties sont distinguées? Quoi qu'il en soit, je crois nécessaire ici d'en rétablir la profonde continuité. Les six contributions qui forment ces deux ensembles ne convoquent en effet que trois nouveaux « héros »: la Vie, l'Histoire, le Savoir.

8 Entre le roman et la vie, les liens sont multiples : le travail fictionnel de l'existence et la dimension référentielle de la fiction s'entretiennent l'un l'autre et se donnent à voir dans la confrontation que suggère Marie-Claude Schapira entre l'Histoire de ma vie et Le Compagnon du Tour de France chez George Sand. De manière plus suggestive encore, elle montre l'affleurement de la «triste vie » dans le roman (p. 76). Et à cette appréciation éthique de la vie correspond un jugement esthétique qui appartient également à ce premier $\mathrm{XIX}^{\mathrm{e}}$ siècle et que la contribution de Nathalie Vincent-Munnia met en évidence. Il y a une beauté poétique de la vie, de la vie nue en particulier, de l'existence minimale du peuple: en témoigne le fait que, dans le roman de Sand, la langue populaire est considérée comme immédiatement poétique. Mieux, et cela mériterait d'être encore mieux mis en valeur; c'est la poésie qui signale le populaire, le "primitif», le « naturel », la vie dans ses dimensions essentielles.

9 De la Vie à l'Histoire, il n'y a qu'un pas que ce volume réalise sans toutefois clairement l'exprimer. L'histoire doit faire « sentir la vie » (Michelet) et, en retour, le tournant des $\mathrm{XVIII}^{\mathrm{e}}$ et $\mathrm{XIX}^{\mathrm{e}}$ siècles correspond au surgissement de l'historicité des sociétés et des individus. L'histoire est dans la vie, et Le Compagnon du Tour de France se fait l'écho de cette historicité neuve : le passé cesse d'être un spectacle pour devenir un milieu. D'où la nécessité, comme pour l'existence individuelle, d'un travail fictionnel spécifique qu'évoque Catherine Mariette-Clot. Cette conjonction de la "vie historique » et du " passé vivant » se retrouve d'une autre manière, dans le cœur du roman, dans ce motif transversal qu'est la restauration de la chapelle. Claudine Grossir établit de manière convaincante en quoi ce chantier est au comte de Villepreux ce que la politique des Monuments historiques est à la Monarchie de Juillet. Sur ce plan, rendre vie aux monuments et au passé peut d'ailleurs faire l'objet de deux stratégies distinctes : l'une, intuitive, que représente ici le personnage d'Amaury et qui concerne la mémoire des gestes et de l'« esprit » (un patrimoine immatériel, dirions-nous aujourd'hui) ; l'autre, plus réflexive, que représente Pierre Huguenin et qui s'applique à l'élaboration d'une cohérence monumentale.

10 Cette opposition entre les deux personnages, qui traverse et articule l'ensemble du roman, gouverne le troisième objet que j'ai identifié malgré (mais je ne crois pas contre) l'économie de l'ouvrage : le rapport au Savoir. L'excellent texte de Claire BarelMoisan rappelle avec clarté que ce rapport est complexe et qu'il n'est pas nécessairement transparent. Mieux, il y a sans doute une part d'obscurité qui doit être ménagée de façon à laisser se déployer la diversité des interprétations, la loi de 
l'élection des meilleurs, le règne de la sanior pars qui saura en déterminer le contenu exact. À cet obscurcissement, qui est l'une des règles de l'initiation - et il est dommage que cette dimension initiatique n'ait pas été mieux convoquée dans l'ordre des savoirs -, il faut ajouter, comme le fait C. Barel-Moisan, l'insistance de G. Sand sur la part «innée », «spontanée » du savoir, et même sur «l'absence du savoir» (p. 99) signalant par là une connaissance qui s'ignore comme telle et qui est, en quelque sorte, le propre du personnage d'Amaury, le tailleur de pierre, l'artiste. Sophie Anne Leterrier prolonge cette analyse: tandis que Pierre Huguenin rappelle la nécessité de l'étude, Amaury incarne la force de l'autodidaxie (p. 138).

11 Le volume s'achève sur l'« accueil de l'œuvre ", qui est ici un accueil contemporain, contextualisé, «historiciste » pour reprendre la terminologie de G. Stocking. Deux angles sont adoptés. L'un développe, par l'intermédiaire de Jean-François Chanet, la façon dont le roman, malgré son auteur parfois, propose une éducation politique du peuple par l'inculcation, non scolaire, d'une foi dans le dogme républicain. Le Compagnon devait édifier, mais l'on a trop peu d'élément pour mesurer son impact réel à ce niveau. Par contre, les données sont plus fournies quand il s'agit, sous la plume de Martine Watrelot, de présenter la diffusion du roman en Europe et la place qu'il occupe au sein de cette "littérature de la voix» dont M. Watrelot n'ignore rien. La requalification du roman comme «fiction ethnographique » (p. 157) est intéressante et invite à des prolongements. Ne peut-on, en effet, établir un lien entre l'écriture du roman et la multiplication des enquêtes sur les parlers, les savoirs et les coutumes populaires des «pays » de France depuis celles, inaugurales, menées au début du siècle par l'Académie celtique?

Il ne faut pas se méprendre. Les interrogations et les remarques ici proposées ne reflètent aucunement une insuffisance du volume; elles sont bien davantage le symptôme de son potentiel suggestif et de son caractère stimulant. Les quelques regrets exprimés, ici et là, n'enlèvent rien à la bonne qualité générale de l'ouvrage qu'augmentent de façon heureuse plusieurs index. 